\title{
The 2015 Nobel Prize for medicine: is hope becom- ing a reality for treating neglected African diseases?
}

by Chiara Castellani*

\author{
*M.D., Ph.D., M.P.H. BDOM Kenge, Dem. Rep. of Congo; e-mail: bdomkenge@gmail.com
}

This year the Nobel Assembly at Karolinska Institute decided for the first time to award the Nobel Prize in Medicine to three researchers finding new treatments for neglected tropical diseases (NTD). The neglected tropical diseases are so called because pharmaceutical industry failed to devote sufficient attention towards finding effective cures and treatments for conditions that account for a huge amount of the global disease burden. NTD are a diverse group of communicable diseases that prevail in tropical subtropical conditions affecting more than one billion people who are mainly living in poverty, without adequate sanitation and in close contact with infectious vectors and domestic animals and livestock (1).

William Campbell and Satoshi Omura were awarded for their work on avermectin, a compound active against roundworm, particularly river blindness (onchocerciasis) and lymphatic filariasis, diseases that cause immense suffering and severe disfigurement. The third Nobel prize co-winner was the Chinese pharmacologist Youyou Tu awarded for the discovery of artemisinin, which led to the artemisinin-based combination treatments (ACTs) against malaria. All these diseases are prevalent in low income countries, particularly in sub-Saharian and equatorial Africa.

This Nobel Prize in Medicine is important because rolls out treatments for parasitic diseases. Parasites cause devastating diseases affecting the world's poorest populations, also affected by the consequences of neglected wars, and absent or scarce public healthcare (2). All these problems represent a huge barrier to improving human health and wellbeing. Moreover, the availability of existing effective drugs and care is not enough when the vicious circle of poverty-war-poverty is not interrupted. I am working in a poor area of Africa, the Bandundu (Figure 1) where another parasitic disease, the African trypanosomiasis (sleeping sickness), is endemic causing severe neurologic disability and death. Eflornithine is a drug effective against African trypanosomiasis and registered for the treatment of sleeping sickness (3). However, the production of eflornithine was stopped in 1995 because it didn't make a profit in its main market, the African countries. Nevertheless, when it was discovered that eflornithine was effective with topical application for women facial hirsutism it was launched on the American market as a cosmetic cream (Vaniqa ${ }^{\circledR}$ ). Only after years of international pressure, the production of eflornithine as a systemic drug restarted in 2001, and the drug was given to the WHO and distributed by non-governative organizations in countries where the sleeping sickness is endemic. It was recently estimated that parasitic diseases cause 534,000 deaths per year and 56.6 million daily lost. Yet, the

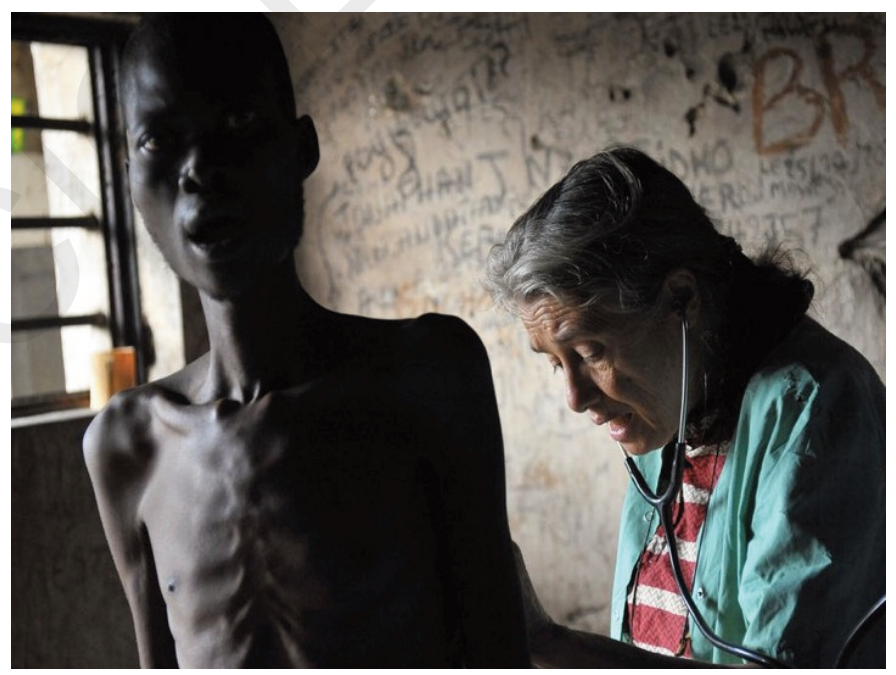

Figure 1 - The Author at work in Bandundu, Democratic Republic of Congo. 


\section{Castellani}

true burden of the NTD is likely to be much higher because of failing comprehensive epidemiological data, overall in the poorest areas of Africa (4). Moreover, there is a geographic overlap of the NTD, which emphasizes that co-infections are pervasive. The available cross-sectional surveys confirmed that polyparasitism is the norm rather than the exception in several countries affected by NTDs. In fact, it has been widely recognized that there is a crosstalk between tropical diseases and malaria, worsening clinical the outcomes of malaria.

After decades of limited progress in developing durable therapies for parasitic diseases, the discoveries by this year's Laureates changed radically the situation. Satoshi Omura, a Japanese microbiologist, isolating new strain of Streptomyces from soil showed its activity against harmful microorganisms. Afterwards, William C. Campbell, an expert in parasite biology working at Drew University in New Jersey, acquired Omura's Streptomyces cultures and purified Avermectin, which was subsequently chemically modified to a more effective and safer compound called Ivermectin. Ivermectin was later tested in humans with parasitic infections and effectively killed parasite larvae (microfilaria). Collectively, Omura and Campbell's contributions led to the discovery of a new class of drugs with extraordinary efficacy against parasitic diseases. Ivermectin has transformed the lives of millions of people suffering from onchocerciasis since 1987 when it was discovered. Onchocerciasis (river blindness) is caused by Onchocerca volvolus worms that lives in the subcutaneous tissue of an infected person. The disease causes intense itching, skin discolouration, rashes, and eye disease, which often leads to blindness. It is spread by infected black flies which breed in rivers. In the Democratic Republic of Congo, the river blindness is still a major health problem for social and economic reasons (Figure 2), but it could be worse without the pioneering researches by Omura and Campbell. But sadly these important advances were exceptions, particularly for poverty-related diseases where commercial incentives for research are not sustainable.

The other half Nobel recipient Dr. Youyou Tu is credited for the discovery of one of the most effective treatments for malaria. Tu and her team trawled through more than 2,000 Chinese remedies for clues on how to fight malaria. One recipe, written 1,600 years ago and entitled "Emergency Prescriptions Kept Up One's Sleeve" proved crucial. It described how sweet wormwood, or Artemisia annua, should be prepared in water to treat the disease. Also in the Equatorial Africa, the beneficial effects of the Artemisia's plant were well known from a long time by the traditional medicine, but is the combined use of artemisinin with other anti-malaric drugs that make it able to block effectively the malaria's plasmodium vital cycle, clearing the parasites from the patient's blood (5). Dr. Tu was able to get support for her discovery from international and national research institutions, and forged connections with key international pharmaceutical companies. They in turn partnered with new, not-for-profit research foundations to ensure the development of efficacious compounds that met World Health Organisation recommendations for adults and children. Political will eventually coalesced, resulting in a twentyfold increase in malaria funding from 2000 until 2015. To date, artemisininbased antimalarials have been delivered to more than 600 million people.

The discoveries recognized by the 2015 Nobel Prize for medicine are perfect examples of the importance to develop novel drugs for NTDs showing what happens when we work together for patients, not just profits. When we will be able to stop the vicious circle of war and poverty we will win the final battle against the neglected tropical diseases.

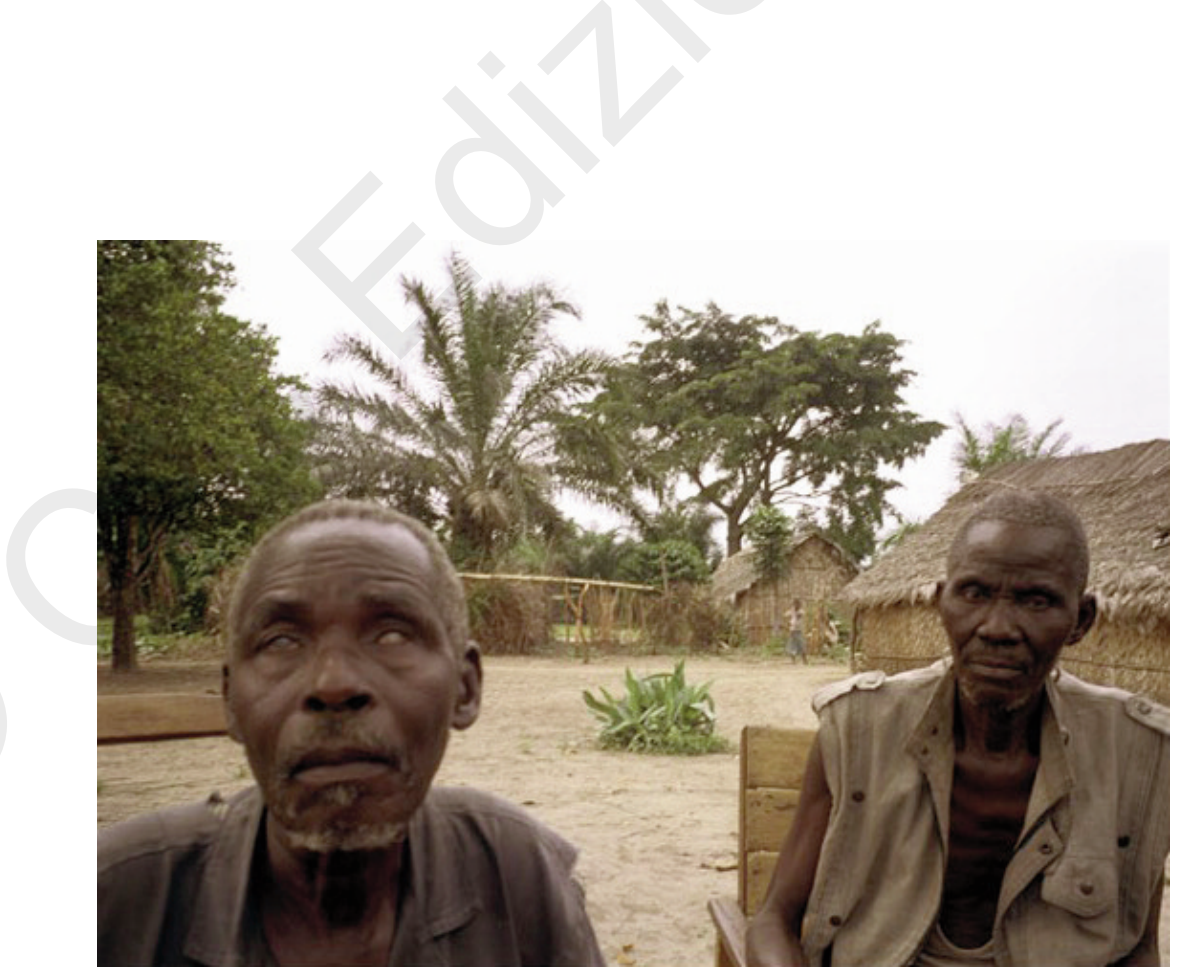

Figure 2 - Patients affected by river blindness in Bandundu, Democratic Republic of Congo. 
The 2015 Nobel Prize for medicine: is hope becoming a reality for treating neglected African diseases?

\section{References}

1. Savioli L, Daumiere D. Accelerating work to overcome the global impact of neglected tropical diseases. A roadmap for implementation. WHO Press 2012, Geneva.

2. Coghlan B, Brennan R, Ngoy P, et al. Mortality in the Democratic Republic of Congo: a nationwide survey. Lancet. 2006;367:44-51.

3. Pepin J, Milord F, Guern C, Schechter PJ. Difluoromethylornithine for arseno-resistant Trypanosoma brucei gambiense sleeping sickness. Lancet. 1987;2(8573):1431-2.

4. World Health Organisation. Disease of poverty and the 10/90 gap. McGuru LTD, London, 2004.

5. Miller LH, Su X. Artesiminin: discovery from the Chinese herbal garden. Cell. 2011;146:855-8. 